\title{
Is the concomitant use of clopidogrel and Proton Pump Inhibitors still associated with increased adverse cardiovascular outcomes following coronary angioplasty?: a
} systematic review and meta-analysis of recently published studies (2012 - 2016)

\author{
Pravesh Kumar Bundhun ${ }^{1}$, Abhishek Rishikesh Teeluck², Akash Bhurtu² and Wei-Qiang Huang ${ }^{1 *}$
}

\begin{abstract}
Background: Controversies were previously observed with the concomitant use of clopidogrel and Proton Pump Inhibitors (PPIs), especially omeprazole, following coronary angioplasty. Even though several studies showed no interaction between clopidogrel and PPIs, questions have been raised about the decrease in antiplatelet effects of clopidogrel with PPIs. A previously published meta-analysis showed concomitant use of clopidogrel and PPIs to be associated with higher adverse cardiovascular outcomes. However, data which were used were extracted from studies published before the year 2012. Whether these controversies still exist in this new era is not clear. Therefore, we aim to show if the concomitant use of clopidogrel and PPIs is still associated with higher adverse outcomes following Percutaneous Coronary Intervention (PCI) using data obtained from recently published studies (2012 to 2016).

Methods: Electronic databases were searched for recent publications (2012-2016) comparing (clopidogrel plus PPIs) versus clopidogrel alone following PCl. Adverse cardiovascular outcomes were considered as the clinical endpoints. Odds Ratios (OR) with 95\% Confidence Intervals (Cl) were used as the statistical parameters and the pooled analyses were performed with RevMan 5.3 software.

Results: Eleven studies with a total number of 84,729 patients (29,235 patients from the PPIs group versus 55,494 patients from the non-PPIs group) were included. Results of this analysis showed that short term mortality and Target Vessel Revascularization (TVR) significantly favored the non-PPIs group with OR: 1.55; 95\% Cl: 1.43-1.68, $P<0.00001$ and OR: 1.26; 95\% Cl: 1.06-1.49, $P=0.009$ respectively. Long-term Major Adverse Cardiac Events (MACEs), Myocardial Infarction (MI), Stent Thrombosis (ST) and TVR significantly favored patients who did not use PPIs with OR: 1.37; 95\% Cl: 1.23-1.53, $P<0.00001$, OR: 1.41; 95\% Cl: 1.26-1.57, $P<0.00001$ and OR: 1.38; 95\% Cl: 1.13-1.70, $P=0.002$ and OR: 1.28; $95 \% \mathrm{Cl}: 1.01-1.61, P=0.04$ respectively. However, the result for long term mortality was not statistically significant. Conclusion: The combined use of clopidogrel with PPIs is still associated with significantly higher adverse cardiovascular events such as MACEs, ST and MI following PCI supporting results of the previously published meta-analysis. However, long-term mortality is not statistically significant warranting further analysis with randomized patients.
\end{abstract}

Keywords: Proton pump inhibitors, Clopidogrel, Percutaneous coronary intervention, Major adverse cardiac events

\footnotetext{
*Correspondence: huangwq1029@126.com

'Institute of Cardiovascular Diseases, the First Affiliated Hospital of Guangxi

Medical University, Nanning, Guangxi 530021, People's Republic of China

Full list of author information is available at the end of the article
} 


\section{Background}

Controversies still exist with the concomitant use of clopidogrel, one of the components of the Dual AntiPlatelet Therapy (DAPT) with Proton Pump Inhibitors (PPIs), especially omeprazole following Percutaneous Coronary Intervention (PCI). Even if the American College of Cardiology/Gastroenterology and the American Heart Association recommend prophylactic treatment with a PPI in those patients who require DAPT and those patients who are at high risk of gastrointestinal injury [1], recent studies have shown clopidogrel and PPIs to be metabolized by the same cytochrome P450 2C19 (CYP2C19) pathway [2].

Several studies showed no interaction between clopidogrel and PPIs. For example, Rassen et al. showed a slight increase in the rate of Myocardial Infarction (MI) and mortality in older patients discharged on clopidogrel and PPIs, but the authors were not able to conclude any interaction between PPIs and clopidogrel in terms of major clinical relevance [3]. Zairis et al. also showed no impact of omeprazole on the clinical efficacy of clopidogrel during the first year following PCI [4].

However, decrease in antiplatelet effects of clopidogrel with the concomitant use of PPIs has been observed. Patients had a higher level of platelet reactivity which resulted in an increased risk of adverse clinical outcomes [5]. For example, Gupta et al. concluded that the concomitant use of clopidogrel with PPIs following coronary stents implantation was associated with a significantly higher risk of major adverse cardiac events (MACEs) [6].

In 2012, Huang et al. conducted a meta-analysis based on the current idea, using old data (2009-2011) [7]. Results from their meta-analysis showed significantly increased risk of MACEs in patients with the concomitant use of clopidogrel and PPIs. Unfortunately, the high level of heterogeneity observed among the different subgroups analyzed was their major limitation.

Recently, many new studies were published based on the cardiovascular outcomes observed in patients treated with clopidogrel plus PPIs and clopidogrel alone following PCI. However, whether these controversies still exist in this new era is not clear. Therefore, we aim to show if the concomitant use of clopidogrel and PPIs is still associated with higher adverse outcomes following PCI using data obtained from recently published studies (2012 to 2016).

\section{Methods}

Data sources and search strategy

Three reviewers (P.K.B, A.R.T and A.B) carefully searched EMBASE, PubMed/Medline databases, and the Cochrane library for Randomized Controlled Trials (RCTs) and observational studies comparing the concomitant use clopidogrel with PPI and clopidogrel alone following PCI. The terms 'proton pump inhibitor and clopidogrel', 'proton pump inhibitor and percutaneous coronary intervention' and 'proton pump inhibitor and dual antiplatelet therapy' were searched carefully. In addition, abbreviations such as PPI, PCI and DAPT were also used. In order to widen the search process, individual PPIs namely 'omeprazole, pantoprazole, lansoprazole, esomeprazole, and rabeprazole' were also used in this search strategy. Because this current meta-analysis was based on recently published English articles, and since the previously published meta-analysis already included old data published before or in the year 2011, only studies published after the year 2011 (2012 to 2016) were considered relevant. Unpublished data were not included.

\section{Inclusion and exclusion criteria}

RCTs and observational studies were included if:

(a) They compared patients treated with (clopidogrel and PPIs) and patients treated with clopidogrel but without PPIs following coronary stenting.

(b) Adverse cardiovascular outcomes were reported as their clinical endpoints.

(c) They were published after the year 2011.

RCTs and observational studies were excluded if:

(a) They did not compare patients (clopidogrel and PPIs) with clopidogrel alone following coronary stenting.

(b)Adverse cardiovascular outcomes were not reported as their clinical endpoints.

(c) They were published before or in the year 2011 .

(d)They were duplicates.

\section{Outcomes and follow up periods}

Reported outcomes which have been listed in Table 1 included:

(a) All-cause mortality

(b) MI

(c) Target vessel revascularization (TVR)

(d)Stent thrombosis (ST)

(e) MACEs which consisted of death, MI and repeated revascularization.

Follow up period was divided into a short term follow up period ( $<1$ year) and a long term follow up period ( $\geq 1$ year).

\section{Data extraction and quality assessment}

Three authors (P.K.B, A.R.T and A.B) independently reviewed the data extracted from the studies included in this meta-analysis. Information regarding the type of study, the total number of patients in the study group and the control group respectively, data regarding the baseline characteristics of the patients involved, 
Table 1 Reported outcomes and their follow up periods

\begin{tabular}{llll}
\hline Study & Reported outcomes & Follow up periods & Type of follow up \\
\hline Bhurke 2012 [17] & Ml and revascularization & 9 months & Short term \\
Burkard 2012 [18] & Death, MI, ST, MACE, TVR & 3 years & Long term \\
Chitose 2012 [19] & Death, Ml & 18 months & Long term \\
Douglas 2012 [20] & Death, Ml & 10 months & Short term \\
Dunn 2013 [21] & Death, MI, TVR & 1 month & Short term \\
Goodman 2012 [22] & Death, Ml, ST & 1 year & Long term \\
Hsieh 2015 [23] & Ml, revascularization & 1 year & Long term \\
Macaione 2012 [24] & Death, MI, TVR & 3 years & Long term \\
Weisz 2015 [25] & Death, MACEs, MI, ST, TVR & In hospital, 2 years & Short and long term \\
Zou 2014 [26] & Death, MACEs, MI, ST, TVR & 1 year & Long term \\
Gargiolo 2016 [16] & Death, MACEs, MI, ST & 2 years & Long term \\
\hline
\end{tabular}

Abbreviations: MI Myocardial infarction, ST Stent thrombosis, MACEs Major adverse cardiac events, TVR Target vessel revascularization

information regarding the cardiovascular outcomes reported as well as the follow up periods associated with each eligible study were systematically extracted. At a certain point, when the authors disagreed about including certain studies, disagreements were resolved and a final decision was made by the fourth author (W.Q.H). Since only two trials were included in this meta-analysis whereas the other studies were observational cohorts, the risk of bias was not assessed [8].

\section{Methodological quality and statistical analysis}

Recommendations from the Preferred Reporting Items for Systematic Reviews and Meta-Analyses guideline were followed [9]. Heterogeneity was assessed using the following:

(a) Cochrane Q-statistic test based on a $P$ value with a cut-off point of 0.05 whereby a value less or equal to 0.05 was considered statistically significant.

(b) $\mathrm{I}^{2}$-statistic test whereby an increasing value denoted an increasing heterogeneity.

A fixed effects model $\left(\mathrm{I}^{2}<50 \%\right)$ or a random effects model $\left(\mathrm{I}^{2}>50 \%\right)$ was used based on the value of $\mathrm{I}^{2}$ obtained.

Odds Ratios (OR) with 95\% Confidence Intervals (CIs) were calculated. The pooled analyses were performed with RevMan 5.3 software.

Publication bias was assessed by observing funnel plots. The reason for using funnel plots was the fact that studies with a smaller volume were used. For studies of smaller volumes, due to the higher degree of random changes, they have a wider distribution of results compared to studies of greater volumes. This might cause asymmetry in the funnels whereby publication bias could therefore be visually estimated.

Ethical approval was not necessary for such types of research articles.

\section{Results}

\section{Study selection}

A total number of 1153 articles were obtained from the searched databases. One thousand and ninety-six articles were rejected since they were either not related to this current topic or they were duplicates. Fifty-seven full text articles were assessed for eligibility. A further six articles were eliminated since they were case studies and meta-analyses. Three more articles were eliminated because their data could not be used (outcomes were reported in terms of Hazard Ratio which was not appropriate to be used in meta-analysis). In addition, 37 more articles were eliminated since they were published before the year 2012. Finally, 11 articles were included in this analysis (Fig. 1).

\section{Baseline characteristics}

A total number of 84,729 patients were included in this analysis $(29,235$ patients treated with clopidogrel plus PPIs and 55,494 patients treated with clopidogrel alone). The general features of the studies have been summarized in Table 2.

Study Douglas 2012, which was conducted in United Kingdom, consisted of the highest number of patients, followed by the studies Bhurke 2012, Dunn 2013 and Goodman 2012 respectively.

The baseline features of the patients have been listed in Tables 3 and 4 lists the different types of PPIs used by the patients.

According to the baseline features, there was no significant difference among the patients who were treated with (clopidogrel plus PPIs) and clopidogrel alone.

\section{Main results of this meta-analysis}

Results of this analysis (summarized in Table 5) showed that during a short term follow up period, using a fixed effects model, mortality and TVR significantly favored 


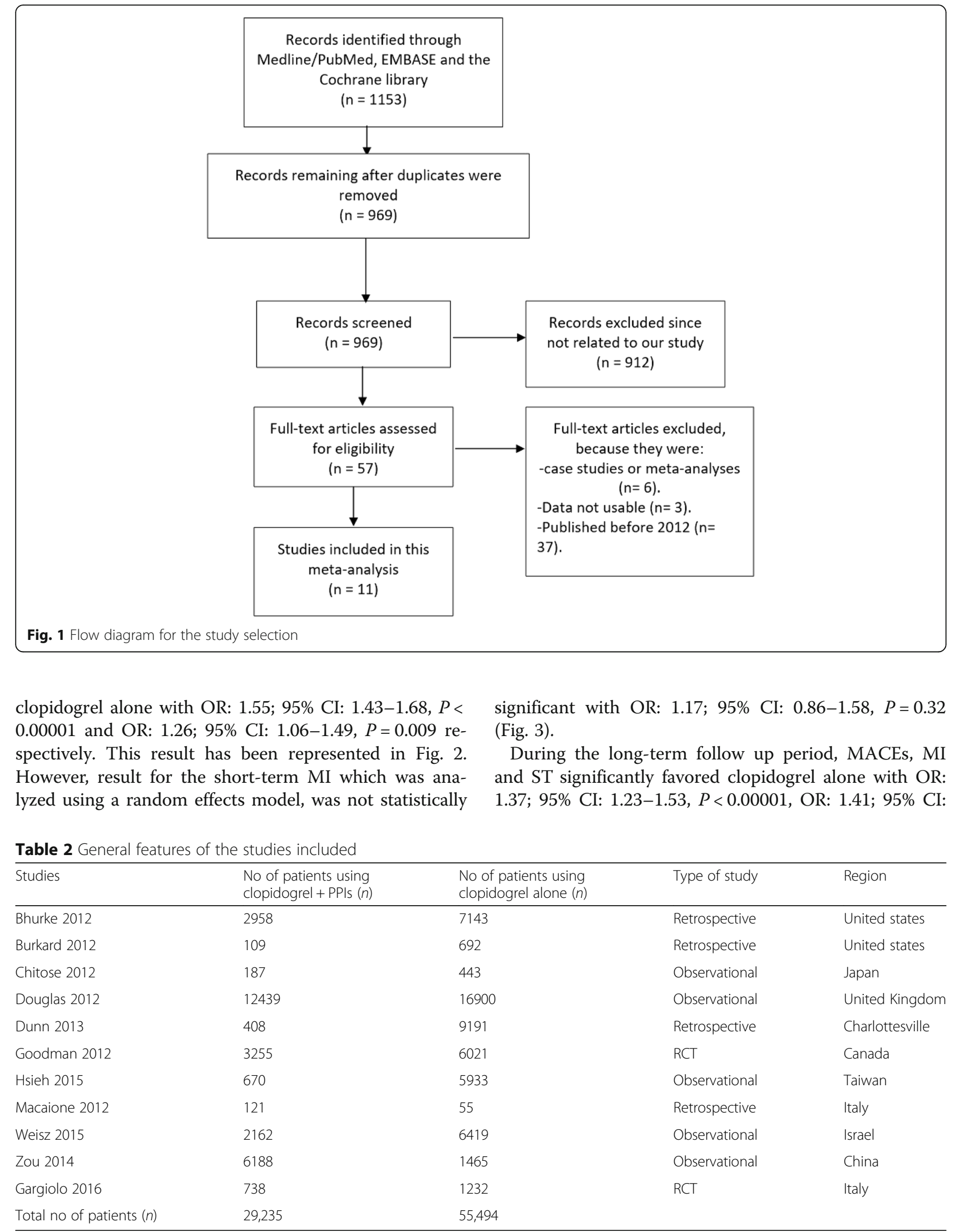


Table 3 Baseline characteristics of the patients

\begin{tabular}{|c|c|c|c|c|c|c|}
\hline \multirow[t]{2}{*}{ Study } & Age (year) & Males (\%) & HT (\%) & Ds (\%) & DM (\%) & CS (\%) \\
\hline & C + PPI/C alone & $\mathrm{C}+\mathrm{PPI} / \mathrm{C}$ alone & C+PPI/C alone & $\mathrm{C}+\mathrm{PPI} / \mathrm{C}$ alone & $\mathrm{C}+\mathrm{PPI} / \mathrm{C}$ alone & $\mathrm{C}+\mathrm{PPI} / \mathrm{C}$ alone \\
\hline Bhurke 2012 & $61.5 / 59.6$ & $68.7 / 76.3$ & - & - & 28.9/26.4 & - \\
\hline Burkard 2012 & $66.5 / 63.3$ & $68.8 / 79.9$ & $72.5 / 65.0$ & 73.4/75.9 & 29.6/17.2 & 24.8/29.8 \\
\hline Chitose 2012 & $70.3 / 68.9$ & 71.6/72.2 & 77.9/79.0 & 61.9/61.7 & $35.3 / 33.7$ & 23.9/26.2 \\
\hline Douglas 2012 & $71.0 / 68.0$ & $58.0 / 65.0$ & - & - & $34.0 / 29.0$ & 16.0/18.0 \\
\hline Dunn 2013 & $63.9 / 62.5$ & $69.3 / 72.3$ & $49.5 / 51.5$ & $39.4 / 41.2$ & $16.5 / 20.3$ & 23.9/29.6 \\
\hline Goodman 2012 & $63.0 / 62.0$ & $72.4 / 71.2$ & $65.6 / 65.4$ & $49.8 / 45.0$ & $25.8 / 24.7$ & $36.2 / 35.7$ \\
\hline Hsieh 2015 & $68.4 / 66.5$ & 63.6/66.4 & - & - & - & - \\
\hline Macaione 2012 & $63.7 / 65.8$ & $80.2 / 87.3$ & $70.2 / 81.8$ & $53.7 / 58.2$ & $41.3 / 49.1$ & $37.2 / 27.3$ \\
\hline Weisz 2015 & $64.4 / 63.2$ & $70.1 / 75.9$ & $83.7 / 77.8$ & $76.9 / 73.2$ & $34.8 / 31.4$ & $22.7 / 22.6$ \\
\hline Zou 2014 & $66.2 / 65.7$ & $73.5 / 73.9$ & $71.3 / 70.4$ & $60.2 / 62.3$ & $25.8 / 23.6$ & $32.2 / 31.0$ \\
\hline Gargiolo 2016 & $71.2 / 68.1$ & $72.5 / 79.2$ & $72.5 / 71.3$ & $53.8 / 55.3$ & $23.3 / 24.8$ & $22.6 / 24.4$ \\
\hline
\end{tabular}

Abbreviations: C Clopidogrel, PPI Proton pump inhibitor, HT Hypertension, Ds Dyslipidemia, DM Diabetes mellitus, CS Current smoker

1.26-1.57, $P<0.00001$ and OR: $1.38 ; 95 \%$ CI: $1.13-1.70$, $P=0.002$ respectively (Fig. 4 ).

However, since a high level of heterogeneity was observed when analyzing the long-term mortality and TVR, a random effects model was used. Long term TVR also significantly favored the non-PPI group with OR: 1.28; 95\% CI: $1.01-1.61, P=0.04$ whereas the result for the long-term mortality was not statistically significant with OR: 1.26; 95\% CI: 0.99-1.60, $P=0.06$ (Fig. 5).

Based on a visual inspection of the funnel plot, there has been a low evidence of publication bias among the studies that assessed several subgroups of adverse cardiovascular endpoints. These funnel plots have been illustrated in Fig. 6a and b.

\section{Discussion}

Controversies still exist with the concomitant use of clopidogrel and PPIs following coronary stenting, which remain to be solved in this new era. In this analysis, we aimed to compare the adverse clinical outcomes associated with the concomitant use of clopidogrel and PPIs versus clopidogrel alone following PCI using data obtained from recently published articles (2012-2016).

This current analysis showed that during a short term follow up period, mortality and revascularization were significantly lower in those patients who did not require treatment with PPIs. Moreover, during the long term follow up period, adverse cardiovascular outcomes such as MACEs, ST, MI and TVR significantly favored patients in the non-PPI group. However, result for the long-term mortality was similar manifested in both groups.

The previously published meta-analysis [7] which included 32 studies with publication date before the year 2012 (29 studies published in English and 3 studies published in Chinese), showed the concomitant use of PPI and clopidogrel to be associated with higher MACEs with OR: $1.27,95 \%$ CI: $1.13-1.42$ when a combination of data obtained from randomized trials and observational

Table 4 Types of Proton Pump Inhibitors used by the patients

\begin{tabular}{llllll}
\hline Studies & Omeprazole & Esomeprazole & Lansoprazole & Pantoprazole & Rabeprazole \\
\hline Bhurke 2012 & 27.1 & 23.1 & 17.6 & 25.8 & 25.0 \\
Burkard 2012 & 17.0 & 51.0 & 7.00 & - & - \\
Chitose 2012 & - & - & - & - & 30 \\
Douglas 2012 & - & - & - & 3.03 & - \\
Dunn 2013 & 14.0 & - & 18.1 & 30.1 & - \\
Goodman 2012 & 48.9 & 11.7 & 7.80 & - & -1.60 \\
Hsieh 2015 & - & - & - & 34.7 & - \\
Macaione 2012 & 43.0 & 11.6 & 10.7 & - & - \\
Weisz 2015 & - & - & - & - & - \\
Zou 2014 & - & - & - & 7.60 & 0.5 \\
Gargiolo 2016 & 0.5 & 0.5 & 90.9 &
\end{tabular}

Percentage (\%) has been used to represent these data 
Table 5 Results of the main analysis

\begin{tabular}{llll}
\hline Outcomes analyzed & OR with 95\% Cl & $P$ value & $1^{2}(\%)$ \\
\hline Short term follow up & & & \\
Mortality & $1.55[1.43-1.68]$ & 0.00001 & 0 \\
TVR & $1.26[1.06-1.49]$ & 0.009 & 22 \\
Ml & $1.17[0.86-1.58]$ & 0.32 & 91 \\
Long term follow up & & & \\
MACEs & $1.37[1.23-1.53]$ & 0.00001 & 0 \\
Ml & $1.41[1.26-1.57]$ & 0.00001 & 29 \\
ST & $1.38[1.13-1.70]$ & 0.002 & 0 \\
Mortality & $1.26[0.99-1.60]$ & 0.06 & 61 \\
TVR & $1.28[1.01-1.61]$ & 0.04 & 72 \\
\hline
\end{tabular}

Abbreviations: OR Odds ratio, $\mathrm{Cl}$ Confidence interval, TVR Target vessel revascularization, MI Myocardial infarction, MACEs Major adverse cardiac events, ST Stent thrombosis

studies was used. However, pooling data only from randomized trials did not show any increase risk of MACEs with OR: $0.92,95 \%$ CI: $0.53-1.58 ; P=0.72, \mathrm{I}^{2}=0 \%$. When mortality was analyzed using a random effects model, a significant increase was observed with HR: 1.30, 95\% CI: 0.91-1.86. But when a fixed effects model was used to analyze mortality, no significant increase was observed with clopidogrel plus PPI with OR: 0.92, 95\% CI: 0.82-1.04.

Several reasons have been suggested for such a result. First of all, PPIs involve the same metabolic pathway (mainly CYP2C19 isoenzyme) with that of clopidogrel [10]. In other words, by occupying the same metabolic pathway as clopidogrel, PPIs are expected to reduce the antiplatelet effects of clopidogrel. Because PPIs can act as both, inhibitors and substrates of CYP2C19, patients treated with clopidogrel and PPIs are vulnerable to a reduced effectiveness of clopidogrel. This could in turn result in a higher platelet activity following PCI finally causing an increase in adverse clinical outcomes. Gilard et al. were the first ones to show the interaction of clopidogrel and PPIs [11]. Moreover, PPIs not only showed a high platelet reactivity but also showed an increased inflammatory state due to the rise in the level of interleukins-6 which in turn could increase the occurrence of ischemic events [12]. However, whether PPIs really have an effect on clopidogrel's antiplatelet effect is still being debated.

Similar to the results of this current analysis, many other previously published studies showed that adverse clinical outcomes were significantly increased in the PPIs group. Gupta et al. concluded that the concomitant use of clopidogrel with PPIs was associated with an increased risk of MACEs following PCI [6]. In addition, Ho et al. showed increased risk of adverse outcomes with clopidogrel plus PPIs [13].

However, even if many studies supported these current results, several other studies showed results which were completely different. For example, Rassen et al. showed that although a slight increase in hospitalization due to MI and death was observed in older patients who were prescribed PPIs and clopidogrel together, there was not enough evidence to conclude any major interaction between these 2 drugs [3]. In the analysis from the Guthrie Health Off-Label Stent (GHOST) Investigators, the

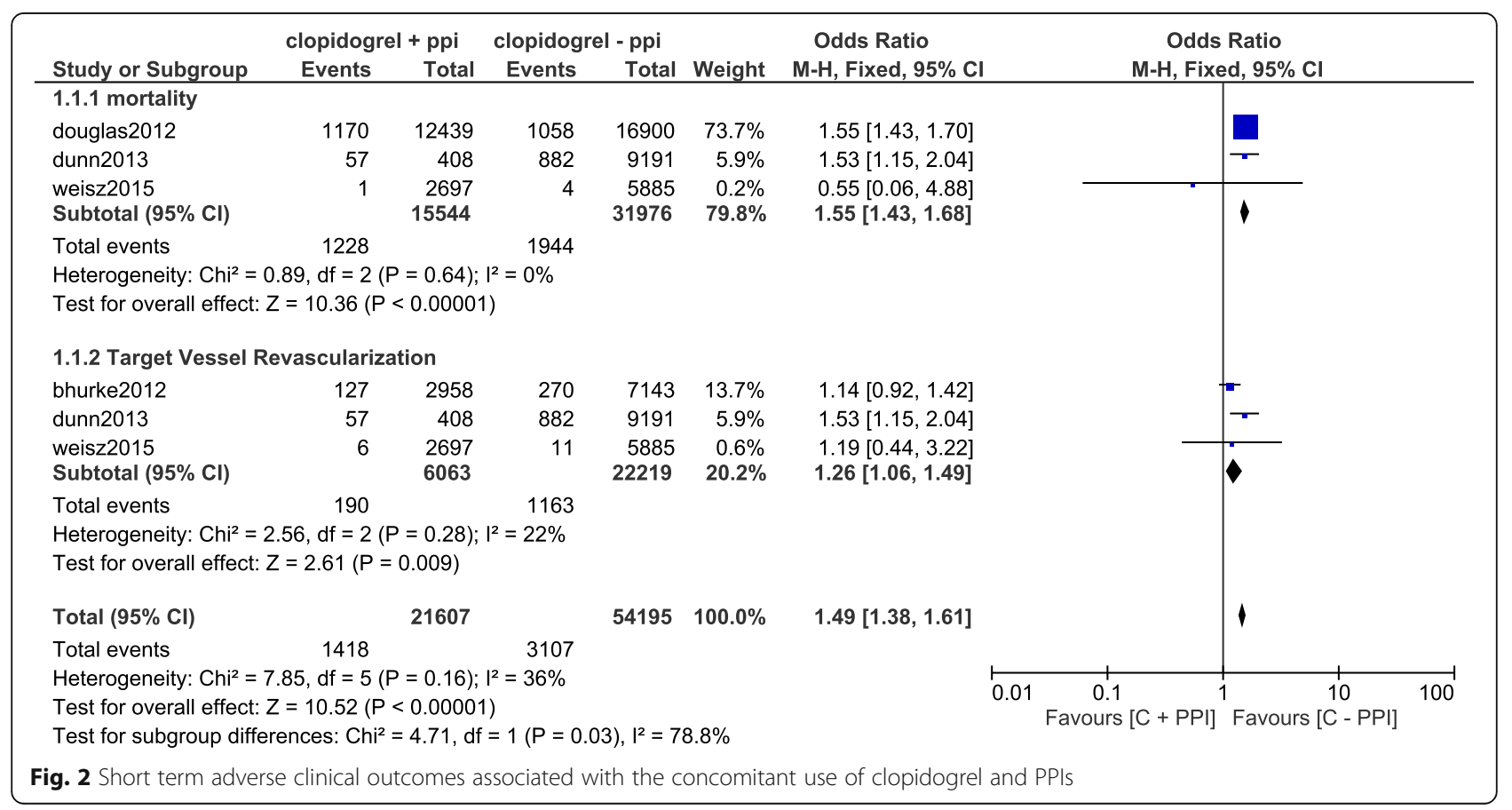




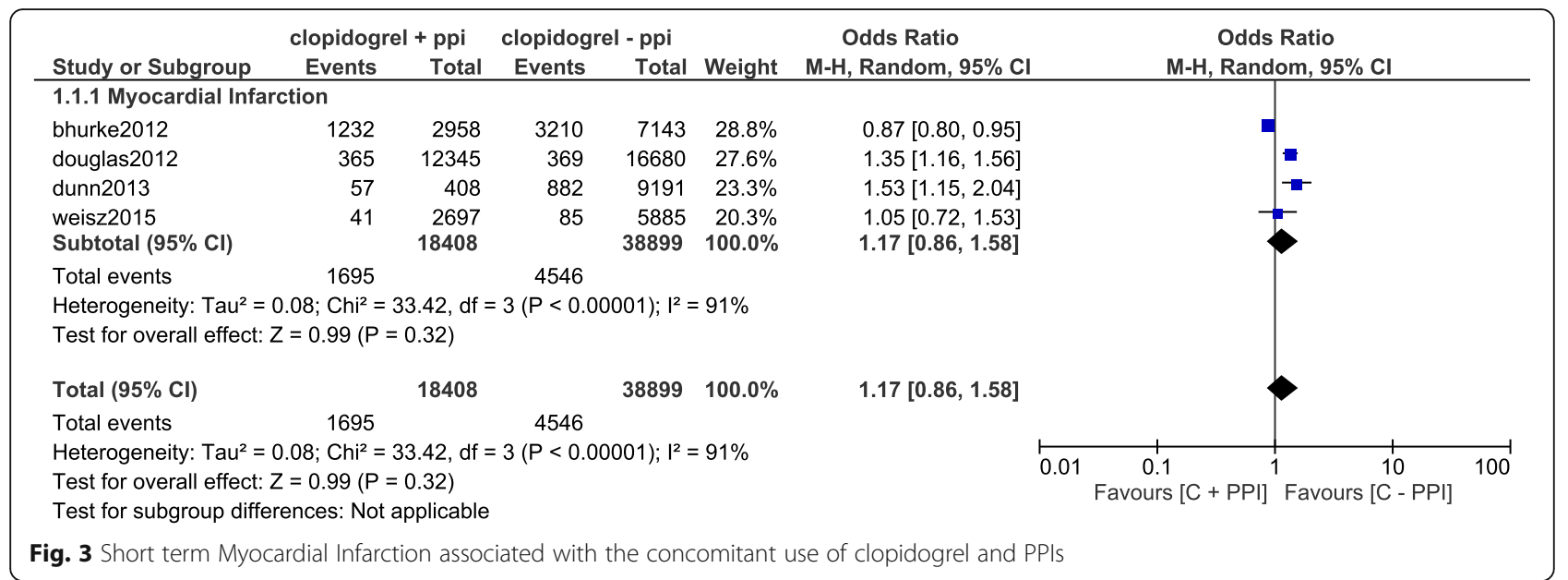

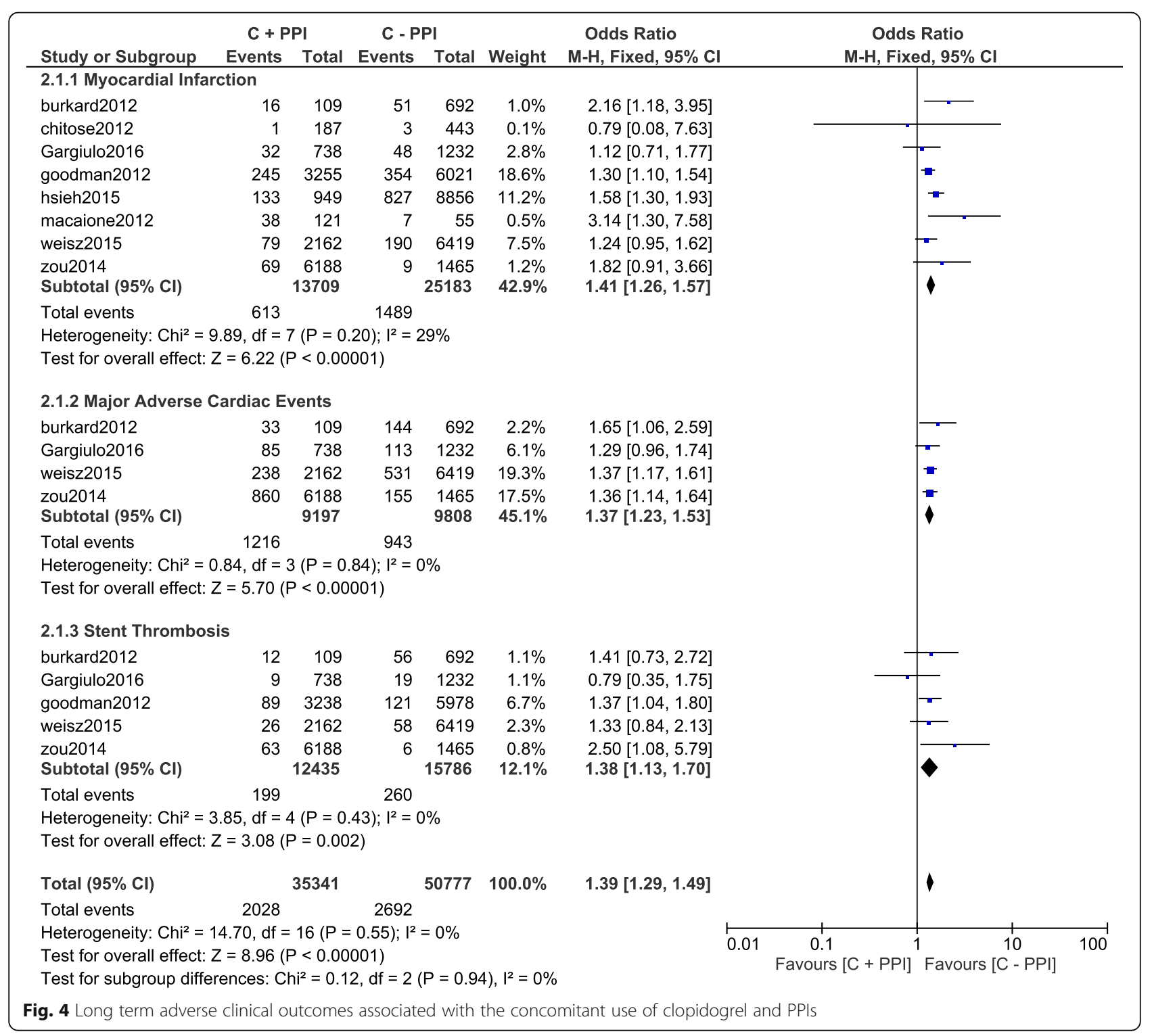




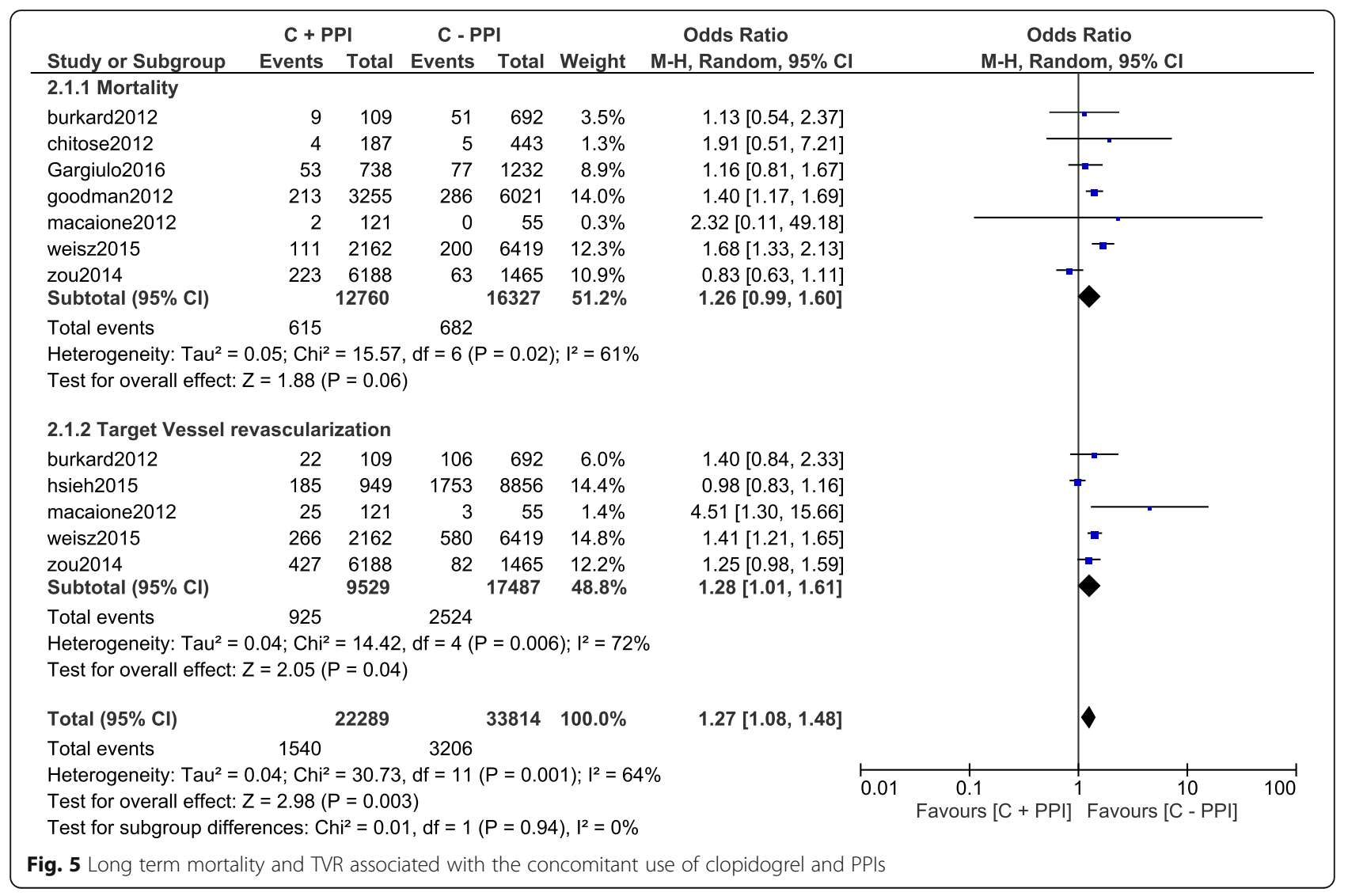

authors also concluded that the use of PPIs with DAPT was not associated with any increase in MACEs following PCI [14]. However, their study had a follow up period of only 6 months. Zairis et al. also showed no impact of omeprazole on the clinical efficacy of clopidogrel during the first year following successful PCI [4]. However, the authors concluded that further highly powered studies should be conducted to confirm whether or not, omeprazole has any effect on the antiplatelet mechanism of clopidogrel. In addition, the COGENT study also did not observe any apparent interaction between clopidogrel and omeprazole, but however, the authors strictly mentioned that their results did not rule out clinically meaningful differences in adverse cardiovascular outcomes due to the use of PPIs [15].

Nevertheless, among all the PPIs, omeprazole is considered to have a higher effect on the mechanism of clopidogrel. Other studies did not show any notable inter-reaction among non-omeprazole PPIs and clopidogrel. For example, when pantoprazole was used along with clopidogrel, no increase in adverse events was observed and therefore pantoprazole has been recommended compared to omeprazole in patients treated with clopidogrel. In addition, in a sub-analysis of the randomized PRODIGY trial, it was reported that the concomitant use of PPIs, when clinically indicated, in patients receiving clopidogrel, was not associated with adverse clinical outcomes. However, it should also be noted that only less than $1.5 \%$ of the patients used omeprazole while more than $90 \%$ of the patients in that particular trial used lansoprazole, suggesting that this type of PPIs might be safer to use with clopidogrel [16].

Since the adverse clinical events associated with nonomeprazole PPIs and clopidogrel have still not clearly been studied, further research is recommended with these individual PPIs (esomeprazole, rabeprazole, lansoprazole, and pantoprazole). In addition, bleeding events especially gastrointestinal bleeding associated with the concomitant use of clopidogrel with these individual PPIs should also be carefully studied.

A moderate level of heterogeneity was observed among certain subgroups analyzing the cardiovascular outcomes. Only English publications were considered, and articles written in other languages were ignored, therefore, a language bias might most probably be present. Moreover, data obtained from conference abstracts and other unpublished studies were not included. However, since most of the data used in this analysis were obtained from observational studies, it could be one of the reasons contributing to the moderate risk of bias observed. In addition, a high level of heterogeneity could also have been due to the fact 

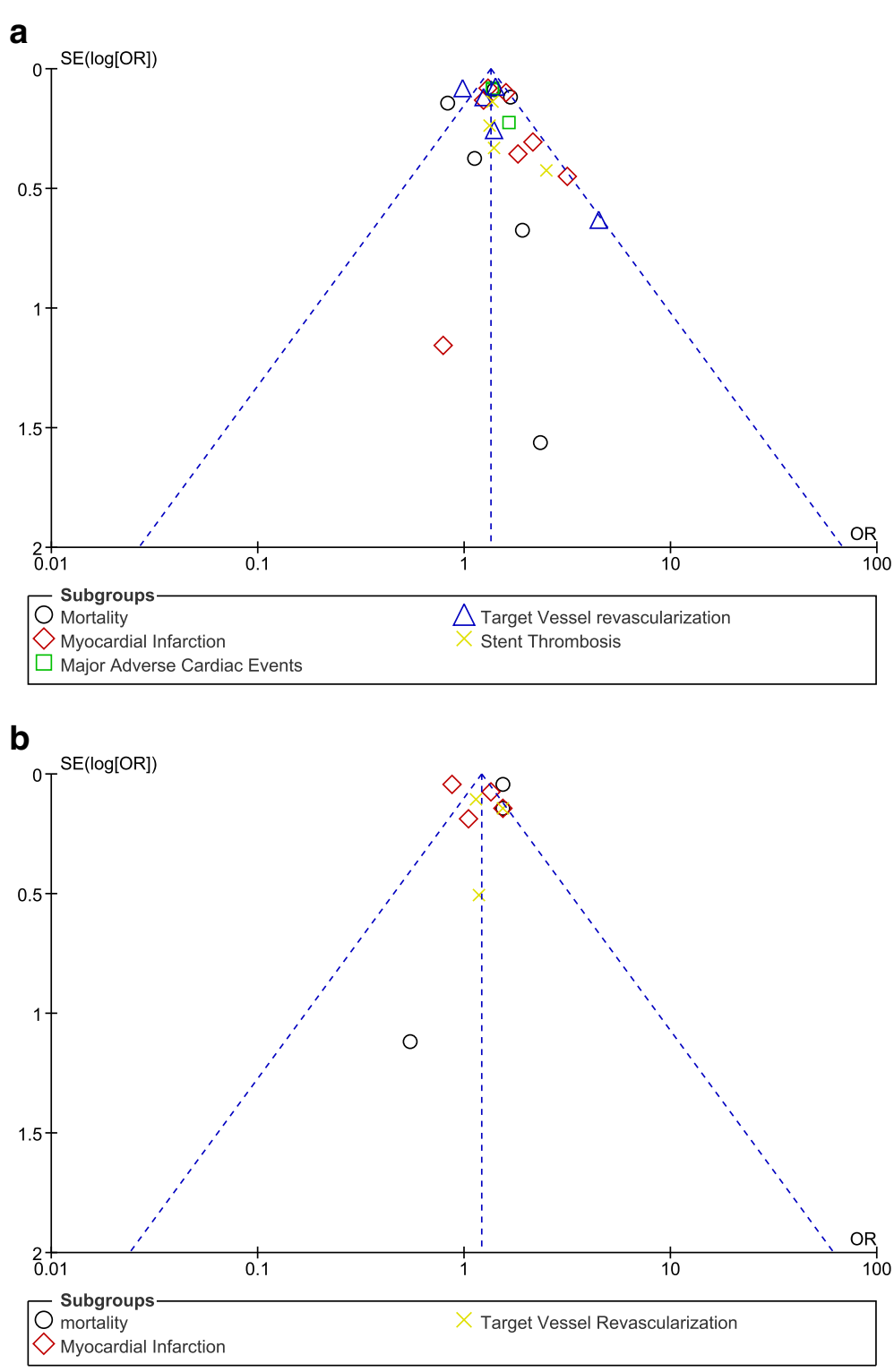

Fig. $\mathbf{6}$ a and $\mathbf{b}$ Funnel plots showing publication bias

that different types of patients were included (chronic stable angina, STEMI, NSTEMI) and the type of stent following PCI was also not taken into consideration; patients implanted with DES and BMS were combined and analyzed.

Novelty in this study is the fact that a lower level of heterogeneity was present among several subgroups compared to the previously published meta-analysis. Moreover, different from other studies which mainly report either short term, mid-term or long term outcomes, this analysis has compared the long term and short term adverse clinical outcomes in patients with and without the concomitant use of clopidogrel and PPIs. In addition, this analysis included data obtained from newly published research articles.

\section{Limitations}

Several limitations are present. Due to a limited number of patients, the result of this analysis might be affected. Moreover, this analysis involved mainly data obtained from observational studies which might be another limitation, and because of this reason, the bias risk of the studies included in this analysis was not assessed using recommendations from the Cochrane Collaboration. In addition, one study reported death, MI and revascularization together. Since data for each outcome could not 
be separated, we have included the same data for this particular study in the different subgroups analyzing mortality, MI and TVR. Also, adverse bleeding events (GI bleeding) were not analyzed because only a few studies reported bleeding outcomes, which were also different in each of the study, making it difficult to compare.

\section{Conclusion}

The combined use of clopidogrel with PPIs is still associated with significantly higher adverse cardiovascular events such as MACEs, ST and MI following PCI supporting results of the previously published meta-analysis. However, long-term mortality is not statistically significant warranting further analysis with randomized patients.

\section{Abbreviations}

DAPT: Dual antiplatelet therapy; MACEs: Major adverse cardiac events;

$\mathrm{PCl}$ : Percutaneous coronary intervention; PPI: Proton pump inhibitor;

ST: Stent thrombosis

\section{Acknowledgements}

Not applicable.

\section{Funding}

This research was supported by the Promotional Project of Guangxi Medical and Health Appropriate Technology (No. S201518)

\section{Availability of data and materials}

All data and materials used in this research are freely available. References have been provided.

\section{Authors' contributions}

PKB, ART, $A B$ and $W Q H$ were responsible for the conception and design, acquisition of data, analysis and interpretation of data, drafting the initial manuscript and revising it critically for important intellectual content. PKB wrote this manuscript. All authors read and approved the final manuscript.

\section{Competing interests}

The authors declare that they have no competing interests.

\section{Consent for publication}

Not applicable.

\section{Ethics approval and consent to participate}

Ethical approval was not applicable for this systematic review and meta-analysis.

\section{Author details}

${ }^{1}$ Institute of Cardiovascular Diseases, the First Affiliated Hospital of Guangxi Medical University, Nanning, Guangxi 530021, People's Republic of China. ${ }^{2}$ Guangxi Medical University, Nanning, Guangxi 530027, People's Republic of China.

Received: 3 November 2016 Accepted: 22 December 2016

Published online: 05 January 2017

\section{References}

1. Abraham NS, Hlatky MA, Antman EM, et al. ACCF/ACG/AHA. ACCF/ACG/ AHA 2010 expert consensus document on the concomitant use of proton pump inhibitors and thienopyridines: a focused update of the ACCF/ACG/ AHA 2008 expert consensus document on reducing thegastrointestinal risks of antiplatelet therapy and NSAID use. A Report of the American College of CardiologyFoundation Task Force on Expert Consensus Documents. J Am Coll Cardiol. 2010;56(24):2051-66.

2. Shah BS, Parmar SA, Mahajan S, Mehta AA. An insight into the interaction between clopidogrel and proton pump inhibitors. Curr Drug Metab. 2012; 13(2):225-35.
3. Rassen JA, Choudhry NK, Avorn J, Schneeweiss S. Cardiovascular outcomes and mortality in patients using clopidogrel with proton pump inhibitors after percutaneous coronary intervention or acute coronary syndrome. Circulation. 2009:120(23):2322-9. doi:10.1161/CIRCULATIONAHA.109.873497.

4. Zairis MN, Tsiaousis GZ, Patsourakos NG, et al. The impact of treatment with omeprazole on the effectiveness of clopidogrel drug therapy during the first yearafter successful coronary stenting. Can J Cardiol. 2010;26(2):e54-7.

5. Gaglia Jr MA, Torguson R, Hanna N, et al. Relation of proton pump inhibitor use after percutaneous coronary intervention with drug-eluting stents to outcomes. Am J Cardiol. 2010;105(6):833-8.

6. Gupta E, Bansal D, Sotos J, Olden K. Risk of adverse clinical outcomes with concomitant use of clopidogrel and proton pump inhibitors followingpercutaneous coronary intervention. Dig Dis Sci. 2010;55(7):1964-8.

7. Huang B, Huang Y, Li Y, et al. Adverse cardiovascular effects of concomitant use of proton pump inhibitors and clopidogrel in patients with coronary artery disease: a systematic review and meta-analysis. Arch Med Res. 2012;43(3):212-24.

8. Higgins JPT, Altman DG. Assessing risk of bias in included studies. In: Higgins JPT, Green S, eds. Cochrane handbook for systematic reviews of interventions. Wiley, 2008:187-241.

9. Liberati A, Altman DG, Tetzlaff J, et al. The PRISMA statement for reporting systematic reviews and meta-analyses of studies that evaluate healthcareinterventions: explanation and elaboration. BMJ. 2009;339:b2700.

10. Lettino M. Inhibition of the antithrombotic effects of clopidogrel by proton pump inhibitors: facts or fancies? Eur J Intern Med. 2010;21(6):484-9.

11. Gilard M, Arnaud B, Cornily JC, et al. Influence of omeprazole on the antiplatelet action of clopidogrel associated with aspirin: the randomized, double-blind OCLA (Omeprazole CLopidogrel Aspirin) study. J Am Coll Cardiol. 2008:51(3):256-60.

12. Jakubowski A, Chlopicki S, Olszanecki R, et al. Endothelial action of thienopyridines and thienopyrimidinones in the isolated guinea pig heart. Prostaglandins Leukot Essent Fatty Acids. 2005;72(2):139-45.

13. Ho PM, Maddox TM, Wang L, et al. Risk of adverse outcomes associated with concomitant use of clopidogrel and proton pump inhibitors followingacute coronary syndrome. JAMA. 2009:301(9):937-44.

14. Harjai KJ, Shenoy C, Orshaw P, Usmani S, Boura J, Mehta RH. Clinical outcomes in patients with the concomitant use of clopidogrel and proton pump inhibitors after percutaneous coronary intervention: an analysis from the Guthrie Health Off-Label Stent (GHOST) investigators. Circ Cardiovasc Interv. 2011;4(2):162-70.

15. Bhatt DL, Cryer BL, Contant CF, et al. COGENT Investigators. Clopidogrel with or without omeprazole in coronary artery disease. N Engl J Med. 2010; 363(20):1909-17.

16. Gargiulo G, Costa F, Ariotti S, et al. Impact of proton pump inhibitors on clinical outcomes in patients treated with a 6- or 24-month dual-antiplatelet therapy duration: Insights from the PROlonging Dual-antiplatelet treatment after Gradingstent-induced Intimal hyperplasia studY trial. Am Heart J. 2016;174:95-102.

17. Bhurke SM, Martin BC, Li C, Franks AM, Bursac Z, Said Q. Effect of the clopidogrel-proton pump inhibitor drug interaction on adverse cardiovascular events in patients with acute coronary syndrome. Pharmacotherapy. 2012;32(9):809-18.

18. Burkard T, Kaiser CA, Brunner-La Rocca H, Osswald S, Pfisterer ME, Jeger RV. BASKET Investigators. Combined clopidogrel and proton pump inhibitor therapy is associated with higher cardiovascular event ratesafter percutaneous coronary intervention;: a report from the BASKET trial. J Intern Med. 2012;271(3):257-63.

19. Chitose T, Hokimoto S, Oshima S, et al. Kumamoto Intervention Conference Study (KICS) Investigators. Clinical outcomes following coronary stenting in Japanese patients treated with and without proton pump inhibitor. Circ J. 2012:76(1):71-8

20. Douglas IJ, Evans SJ, Hingorani AD, et al. Clopidogrel and interaction with proton pump inhibitors: comparison between cohort and within person study designs. BMJ. 2012;345:e4388.

21. Dunn SP, Steinhubl SR, Bauer D, Charnigo RJ, Berger PB, Topol EJ. Impact of proton pump inhibitor therapy on the efficacy of clopidogrel in the CAPRIE and CREDO trials. J Am Heart Assoc. 2013;2(1):e004564.

22. Goodman SG, Clare R, Pieper KS, et al. Platelet Inhibition and Patient Outcomes Trial Investigators. Association of proton pump inhibitor use on cardiovascular outcomes with clopidogrel and ticagrelor: insightsfrom the platelet inhibition and patient outcomes trial. Circulation. 2012;125(8):978-86.

23. Hsieh CF, Huang WF, Chiang YT, Chen CY. Effects of Clopidogrel and Proton Pump Inhibitors on Cardiovascular Events in Patients with Type 2 
DiabetesMellitus after Drug-Eluting Stent Implantation: A Nationwide Cohort Study. PLoS One. 2015;10(8):e0135915.

24. Macaione F, Montaina C, Evola S, Novo G, Novo S. Impact of dual antiplatelet therapy with proton pump inhibitors on the outcome of patients with acute coronarysyndrome undergoing drug-eluting stent implantation. ISRN Cardiol. 2012;2012:692761.

25. Weisz G, Smilowitz NR, Kirtane AJ, et al. Proton Pump Inhibitors, Platelet Reactivity, and Cardiovascular Outcomes After Drug-Eluting Stents inClopidogrel-Treated Patients: The ADAPT-DES Study. Circ Cardiovasc Interv. 2015;8(10):e001952.

26. Zou JJ, Chen SL, Tan J, et al. Increased risk for developing major adverse cardiovascular events in stented Chinese patients treated with dualantiplatelet therapy after concomitant use of the proton pump inhibitor. PLoS One. 2014;9(1):e84985.

Submit your next manuscript to BioMed Central and we will help you at every step:

- We accept pre-submission inquiries

- Our selector tool helps you to find the most relevant journal

- We provide round the clock customer support

- Convenient online submission

- Thorough peer review

- Inclusion in PubMed and all major indexing services

- Maximum visibility for your research

Submit your manuscript at www.biomedcentral.com/submit
Biomed Central 